\title{
Incidence of Measles in Breastfed Infants versus Non-Breastfed Infants
}

\author{
Naim Khan Zadran ${ }^{1}$ and Zabit Khan Naibzi ${ }^{2}$ \\ ${ }^{1 \& 2}$ Pediatric Department, Medical Faculty, Sheikh Zayed University, Khost, Afghanistan. \\ *Correspondence: drnaimkhanzadran123@gmail.com (Dr. Naim Khan Zadran, Lecturer, Pediatric Department, Medical \\ Faculty, Sheikh Zayed University, Khost, Afghanistan).
}

\begin{abstract}
Breast-feeding protects newborn infants against numerous infectious conditions and sicknesses, and can also affect the results of vaccination. The main purpose of this research is to assess breastfeeding defends against $\mathrm{CM}$ and find the changed effects of vaccination. This research is designed as a cross-sectional study. It is worth mentioning that the study took almost one year to be completed, and the survey was conducted from May 1, 2020, to May 1, 2021. It is notable that the study was carried out base on librarian and field research methodology, and the information was collected from the newly born infants until five years' children, and then it was analyzed. The data upon infections of indicative measles and socioeconomic indicators were obtained at ten months' period. Breast-feeding has been classified as < 30 days breastfed, 1-3-month breast-food, breastfeeding for over three months, unsure breastfeeding, and no breastfeeding. Regarding the results, it could be said that anti-measles Ig though with a mean value of 5.37 AFU, all 55 colostrum samples were positive. Furthermore, the timing of extraction for breast milk AFU is different, the average AFU value fell to 2.11 among days 6-15 $(\mathrm{n}=15)$, but the value fell below the cut-out mark of 1.8-1.48 amongst days fifteen to thirty $(\mathrm{n}=10)$. It is being concluded that immunization of measles gives excellent disease resistance. In addition to this, breastfeeding is related to a relatively moderate decrease in the chance of measles diagnosis. Relationships with such a diagnosis of measles were independent of breastfeeding \& measles vaccination.
\end{abstract}

Keywords: Breast-feeding, Measles, Incidence, Vaccination, Breastfed infants, Disease, and Immunization.

\section{INTRODUCTION:}

Measles seems to be a severe disease pathogen, producing around 40 million acute measles infections per year and almost one million infant fatalities. Measles infection has a temporary decrease in children's immunological response, particularly in impoverished places with high infection levels. In 1968, Measles vaccination began 3 in England when kids aged 12 to 15 months were provided with a specific measles vaccine. Regarding 2-year-old children from 1970 and 1980, the covers for vaccinations were about $50 \%$. However, this was 2 insufficient to eradicate measles. For mortality, sickness, and the promotion of a life cycle, infancy remains the most vulnerable stage of human life UniversePG I www.universepg.com
(Campbell, 1983). Worldly 5.3 million fatalities younger than of 5 were projected and improved nursing rates and durability can spare about 800,000 lives. Across Asia, approximately 300,000-350,000 deaths through optimum breastfeeding may be averted, and indeed the preponderance below 12 months would've been prevented (Michelle Science, December 2019).

A comprehensive evaluation of infection and breastfeeding studies is appropriate for children. Breastfeeding protects from numerous infectious illnesses during breastfeeding periods. Emerging findings state that breastfeeding may have long-term protective advantages on immune reaction to vaccinations and con- 
ditions well beyond the actual time of breastfeeding themselves (Shah et al., 2020; Silfverdal, 2009).

The research determines, suppose nursing has longterm beneficial effects from clinical measles infection, or whether monovalent measles immunization has been changed by measles infection (Adu, 1995). Lack of controlled breastfeeding clinical trials implies that breastfeeding research seldom attains the highest level of evidence necessary in the systemic examinations. The main issue with a nursing evaluation is that random treatments are not ethical. The recognized benefits of breastfeeding are to prevent randomization in a control condition of babies.

\section{Measles}

WHO has called for measles removal through universal immunization and controls on the health of human beings? Vaccinations against measles and increased nutrition have resulted in an $80 \%$ and it decrease mortality from 2000 to 2017 in worldwide measles. Measles has averted an incredible 21.1 million deaths over this era. But measles remains a significant cause of 2injury and death in infants. 110,000 fatalities have occurred worldwide, and many other babies and children have been attacked by illness consequences. In several nations, including the very remote Pacific Islands, there has recently been $6 \mathrm{a}$ rise in measles infections. Tonga reported 3728 cases, 53 newborn fatalities this year, with such a populace of just 104 000. A major immunization platform has begun, and 58,000 have been immunized until December 1, 2019. That describes when Europeans navigated the Pacific for the $1^{\text {st }}$ century and ravaged several islands through measles outbreaks. After delivery, circulating maternal antibodies give partial measles immunity so that measles immunization can be postponed until the 1 st year of existence. Breastfeeding provides some measles with coverage, but it takes to be vaccinated just for the most outstanding protection. Protection against babies with possibly positive effects on vaccination responses is provided by the early beginning and special breastfeeding period till six months. Tolerance to other diseases declines following measles illnesses, frequently leading toward a decreasing health cycle. MMR vaccines must be given to vulnerable mothers who breastfed. Throughout the first period, newborns were historically entitled to safety against measles by Tran's placental UniversePG I www.universepg.com acquired antibodies from their mothers. That presumption was based on indication conceded in environments where measles spread endemically. Several women are resistant to natural illness and thus they are subjected to measles continuously, resulting in recurrent immunological stimulation and advanced levels of antibodies. Nevertheless, most women with such a children's childhood age were immune from vaccination within measles-elimination environments. Neither endemic viral transmission lasts for around 12 months and reduces antibody levels than spontaneous diseasing. Moreover, the immunological boosting of vaccine people upon happenstance to measles is significantly decreased, even as measles virus doesn't persist institutionally inside the removal settings. That should lead to babies with lesser titers of Tran's placental antibodies in measles eradication settings falling just below the protective threshold early. Such infants will then be left defenseless to measles to the very first dosage of vaccination that will not be provided until 12-15 months old in eliminating settings. To present, few scientists have exercised the perseverance of measles in children living in removal environments. Motherly antibodies protection, Confidence intervals, emphasizing either newborn immunity or resistance in preterm children, impair the restricted numerous studies done. Investigators have not yet assessed newborn immunity at short distances throughout the first year of existence. Since 1963, the vaccination coverage in Ontario is typically good, and Canada has maintained a systematic measles immunization program. Consequently, in the first six-month female childbirth. We assumed that newborn immunity to measles in our disposal environment would decrease because of the extreme immunity generated by vaccination instead of the normal immunity produced by infection. That is due to the continuous transmission of measles elsewhere globally, which puts babies at hazard for diseases and problems connected to illness.

\section{Symptoms of Measles}

Measles symptoms usually occur within 10 to 12 days of viral contact, including; cough, fever, red-eyes, runny nose, white dots mostly in mouth painful neck. A common skin rash is a characteristics measles symptom. That rash is usually up to seven days after contact to either the infection and occurs within 14 days. It 
usually starts on the face and extends gradually to certain other areas of the body.

\section{Causes of the Measles}

Human Beings are infected by either a paramyxovirus, and the viruses are little microorganisms of parasites. This virus infects host cells after you are contaminated and utilizes cellular functions to continue your cycle of life. That measles virus initially enters the airway and it ultimately shift through the circulation to other parts of the body. Mainly people are known to be extant with measles. There are twenty-four hereditary kinds of metabolisms, which are identified yet, but 6 of these metabolisms are measles and exist. Measles is very infectious, and measles shift and transfer easily from one person to another person. When a person is infected with the measles virus $90 \%$, he/she is vulnerable. It means that when the infection reaches to $90 \%$, it harms the person. Furthermore, around 9-18 vulnerable people may transfer the virus. If you already have measles, you can transfer the virus to someone else before realizing it in four days. They remain infectious for four more days just after the rash appears. Unvaccinated person would be the high-risk factor for measles transmission. Some individuals, notably small children and weaker immune structures and pregnant females have a higher chance of acquiring symptoms of measles infections (Bashar et al., 2020).

\section{Infant measles}

The vaccination against measles is not administered to infants up to one year. It's indeed essential for them to be vulnerable to the measles virus until getting their 1st vaccination dosage. Babies are protected against measles via maternal antibodies from mother to baby via placental and also during nursing. Nevertheless, investigation shows that this immunity may be eliminated in a little over 2.5 weeks after delivery or lactation. The risk of associated infection with measles is greater in children under 5 years. Some illnesses such as; pneumonia, encephalitis and ear infections might lead to hearing loss.

\section{Measles incubation time}

Incubation time is 10 to 14 days during measles. The duration between infection and manifestations develops called the incubation period of such an infectious illness. Patients may extend non-specific indications, including fever, coughing and sneezing, beyond the UniversePG I www.universepg.com initial incubation time. After some days, the rashes will start to emerge. It is crucial to remind that one person may still transfer the illness to others in four days before the rashes develop. Parents must call their doctor urgent if they believe that their child has been infected with measles and have not yet been vaccinated.

\section{Types of Measles}

There are indeed numerous additional forms of measles infections compare to a typical metabolism illness. A typical measles grew from 1963 to 1967 who were vaccinated against measles. Exposure to measles occasionally leads to a disease with indications including high fever, rashes and pneumonia. Enhanced measles grows in individuals who've already received immuneglobulin post-exposure and in passive children. The measles that has been transferred are usually less severe than the ordinary measles. Across the United States, it is seldom known as hemorrhagic measles, that produces symptom such as high fever, convulsions and bleeds into the surface $\&$ membrane of mucus.

\section{Prenatal measles}

Expectant mothers who don't have a measles antibody should be careful not to be exposed during gestation. Measles could have a substantially enlarged risk of complications, mostly on the mother than on the fetus throughout your gestation. The danger of measles results pneumonia, which is greater for expectant mothers. Furthermore, measles during pregnancy may contribute to problems of pregnancy; still birth, Miscarriage, Labour preterm, Weight of low birth. Measles could be readily transferred to the newborn baby when mother has metabolism around her time of delivery. Congenital measles is termed, and congenital measles infants get a rash at delivery or develop soon afterwards. When a female gets German measles during pregnancy, her growing infant might contract the virus via her circulation. Congenital rubella syndrome causes miscarriages but also deaths as well as being a major problem for health (Salam and Aktar, 2020). Newborn, who transported to gestation, can potentially cause birth abnormalities, including: late growth, cardiac defects, intellectual disability, Loss of hearing, Organ malfunction. Ladies of childbirth must be treated before they become pregnant with their resistance against rubella. They must have it at minimum of 28 days before you 
attempt to conception if you require a vaccination. As far as we know, research analysis involved one of the most significant cohorts utilized to help vulnerability of toddlers to measles thru the 1 st year of their lifetime. It is worth noting that until the first vaccination with an initial dosage of measles-containing vaccine, approximately the bulk of newborns are vulnerable to measles till $3^{\text {rd }}$ months of infancy. Too far, research in elimination contexts for newborn vulnerability to measles has focused primarily on preterm babies between the ages of 6 months to 12 months, perhaps on the premise that mom's antibodies shielded toddlers under 6 months. It is expected, because such environments strive to eliminate measles; therefore, immunologically boosting vaccinated moms are probably less frequent than that in high prevalence environments, which leads to reduced antibody concentrations. Numerous lowincidence investigations have indicated that the resistance to measles has decreased even quicker. A virus transmits the breathing difficulties of such a highly infectious anathematic illness for some days during and before the disease. The measles virus still not being identified in breast milk. However, specific antibodies have been discovered in the milk of vaccinated mothers. When measles is diagnosed inside a mother, immunoglobulin should indeed be given to infant and separated from a mother for up to 72 hours after exanthema appears. However, the newborn can feed breast milk as secretory IgA starts to be discharged after 48 hours just after exanthemas manifestation thru the mother. Our research demonstrates that we're on opposite direction, but it is not wonder. Since number of females in our cohort was likely to get immunized more remotely than younger mothers; a century after implementation of the regular vaccination programs, the eldest parents of the generation were born. This increased difference in the loss of mother's antibodies to the immunization for measles reported in this investigation leaves children exposed to metabolic disease during most of infancy. Potential concerns comprise; (1) Baby techniques; (2) Maternity strategies; (3) More comprehensive initiatives for population health. As far as baby methods, now in use in Canada, newborns are vaccinated based on immunological system development with the $1^{\text {st }}$ dosage of measles-containing vaccination, maximizing population security and preventing possible interaction with either the UniversePG I www.universepg.com antibodies during the first year of childhood. Vaccination is postponed upwards to 15 months in several other disposal settings. That leads to a significant susceptibility gap between measles and newborns in removal settings. While early vaccinations are safe and very immunogenic between 6 and 12 months, research suggests that this first dosage around nine months of age may be a dull immune system. It might even be possible to misinterpret and retrieve biases between our data. Infection information was dependent on parental conversations and medical information though not on serology but rather culture confirmations. Parents may recall youngsters infected with such a medical measles infection. These medical records show that individuals with the sickest toddlers will still have medical treatment. We concentrated, although on individuals with clear, usually visual, and clinically apparent symptomatic measles infection.

\section{RESULTS:}

Breast-feeding may not be protective of symptomatic measles but may be linked with unusual manifestations that are as serious as usual conditions but much less probably to be detected. As was the preliminary study, we analyzed how measles infections were evaluated between five to ten years old and not since birth. That excluded that breastfeeding was due simply to such a precautionary character that only worked even during the breastfeeding phase. Starting to obey at65 years (when all breastfeeding was stopped) will result in prejudice that does not hazard later measles detection for those infected earlier. The research would also reduce the possible influence of contemporary effects as a result of childcare attendance discrepancies. Regarding anti-measles Ig Though with a mean value of 5.37 AFU, all 55 colostrum samples were positive. Furthermore, the timing of extraction of breast milk AFU differed. The average AFU value fell to 2.11 among days 6-15 $(n=15)$, but the value fell below the cut-out mark of $1.8-1.48$ among days 15 to $30(n=10)$. The remainder of the analyzed samples $(n=322)$ was off 1.32 mean AFU for 30 days to 1 year. The AFU properties of different AFU levels differed from measurable $(p<0.05)$ samples. Breast milk was found from 12 women that provided colostrum for a subsequent study on transmission of MV-species IgA from colostrum and breast milk. Results of the isolates were 
positive for all 12 mothers with an average AFU value of MV-specific IgA from 1.85 to 15.00 . In contrast, only one of the breast specimens was positive (0.26 to 216).

\section{DISCUSSION:}

Specific diseases and breastfeeding Flavi virus, Ebola, Zika and HIV, hepatitis, cytomegalic virus, HLV1 can transfer various transferable illnesses such as breastfeeding. Brucellosis was observed, too-the WHO keeps a database of illnesses that might be contraindicated for breastfeeding and require special treatment. Not with standing, because of several advantages of breastfeeding, throughout many situations, the perks of breastfeeding might surpass the dangers associated with using formulas, especially in resource-deprived areas. WHO guidance concluding infectious conditions such as; Breast abscess: the unimpaired breast should continue breastfeeding; after therapy has begun feeding, the afflicted breast can continue. Hepatitis B: hepatitis $B$ vaccination must be administered for babies in 2 days or even as promptly as feasible. Tuberculosis: mom and newborn must be treated per national standards for tuberculosis. Because of the rewards of breastfeeding, the overall benefits of breastfeeding are in many situations more considerable than the risks of breastfeeding, sometimes in specific illnesses, particularly among resource-poor settings. Although the WHO has listed several reasons why nursing is not possible, in certain situations, and breastfeeding has continued throughout the decade. For instance, additional research on breast milk trafficking and HIV, HTLV1, and Zika virus incidence is available.

\section{CONCLUSION:}

The evidence is quite obscure when it comes to mom's nursing and non-active immunity. The best point I might offer would be that greater protection is provided by vertical transmission (in-utero immunity transmitted by mother immediately to her baby thru the placenta) than by breastfeeding antigens. We are aware that there's probably immunological protection from measles in breast milk. However, its usefulness is unknown. During the breastfeeding, the kid will not be protected fully from breastfeeding illness. When mother gets immunized 3 due to measles and that she is guarded for a bit longer, or if mother had measles, a full-term kid is generally safeguarded from measles for UniversePG I www.universepg.com approximately a month after delivery. The suggested periods for 2 the MMR vaccination is for the infants for first dosage per the Centers are from 12 to 15 months, for Disease Control \& Prevention (CDC). Second dosage for the youngsters 1between 4 and 6 years old and adults aged 18 years and older \& conceived after 1956 must be given one dosage to confirm that almost all three illnesses or vaccinations were previously present. The Infants from 6 to 112 months, must be vaccinated for the first dosage before travelling overseas. Around age of the 12 months, such infant must continue to receive two doses. The two doses must be received by children at the ages of 12 months and above, before travelling. If anybody reach to twelve months or above, is considered to be at higher risks of having measles throughout an epidemic, then another mumps vaccination must be given. In all situations, at least in 28-days intervals must be administered in the dosages.

\section{ACKNOWLEDGEMENT:}

The authors received no financial support for the research, authorship or publication of this article.

\section{CONFLICTS OF INTEREST:}

The authors declare no conflicts of interest.

\section{REFERENCES:}

1) Adu F D, J. A. (1995). Measles antibodies in the breast milk of nursing mothers. Retrieved June 30, 2021, from -

https://pubmed.ncbi.nlm.nih.gov/8886155/

2) Bashar MA, Haque AKMR, and Rahman R. (2020). Prevalence of anaemia among pregnant women in a rural area of Bangladesh: impact of socio-economic factors, food intake and micronutrient supplementation. Am. J. Pure Appl. Sci., 2(1), 1-7. https://doi.org/10.34104/ajpab.0200107

3) Campbell, A. G. (1983). Measles immunization: why have we failed? Retrieved June 30, 2021, from - https://adc.bmj.com/content/58/1/3

4) Joacy G. Mathias, H. Z.-R. (2019). The association of infant feeding patterns with food allergy symptoms and food allergy in early childhood. Retrieved June 30, 2021, from https://internationalbreastfeedingjournal.biomedcen tral.com/articles/10.1186/s13006-019-0241-x 
5) Joel A. Lamounier, Z. S. (2004). Recommendations for breastfeeding during maternal infections. Retrieved June 30, 2021, from -

https://www.scielo.br/j/jped/a/6G8RsV87xGDZVT MJDZBCnwD/?lang=en

6) Mi Kyung Lee, A. C. (2019). Breastfeeding and the Risk of Infant Illness in Asia: A Review. Retrieved June 30, 2021, from https://www.ncbi.nlm.nih.gov/pmc/articles/PMC69 81475/

7) Michelle Science, R. S. (2019). Measles Antibody Levels in Young Infants. Retrieved June 30, 2021, from -

https://pediatrics.aappublications.org/content/144/6/ $\underline{\mathrm{e} 20190630}$

8) Moyer, M. W. (2015). Does Breast-Feeding Protect My Baby from Measles? Retrieved June 30, 2021, from -

https://slate.com/human-interest/2015/02/measlesand-infants-advice-for-parents-of-unvaccinated-ba bies.html

9) Networks, T. B. (2021). Measles vaccination and breastfeeding. Retrieved June 30, 2021, from https://www.breastfeedingnetwork.org.uk/measles/

10) Salam S., and Aktar B. (2020). Child marriage in rural Bangladesh and its consequences on reproductive and maternal health: an empirical study, Eur. J. Med. Health Sci., 2(4), 74-85. https://doi.org/10.34104/ejmhs.020.074085
11) Shah S, Rana S, Parvin T, and Jalali MA. (2020). Study on awareness, knowledge and practice of breast screening methods among the female students of Bangladesh university of health sciences, Eur. J. Med. Health Sci., 2(6), 152-158. https://doi.org/10.34104/ejmhs.020.01520158

12) Silfverdal SA, A. E. (2009). Breast-feeding and a subsequent 2diagnosis of measles. Retrieved June 30, 2021, from -

https://onlinelibrary.wiley.com/doi/full/10.1111/j.1 651-2227.2008.01180.x

13) Sibylle Schneider-Schaulies, V. T. (2004). Measles virus and immunomodulation: molecular bases and perspectives. Retrieved June 30, 2021, from -

https://www.cambridge.org/core/journals/expert-re views-in-molecular-medicine/article/abs/measles-vi rus-and-immunomodulation-molecular-bases-andperspectives/6746283C7E804989FC6BC34 A66B9 $\underline{720 \mathrm{C}}$

14) Swanson, W. S. (2019). Measles, MMR Vaccine, Immunity, \& Breast milk. Retrieved June 30, 2021, from -

https://seattlemamadoc.seattlechildrens.org/measles -mmr-vaccine-immunity-breastmilk/

15) Wendy Sue Swanson, M. M. (2019). Measles, MMR Vaccine, Immunity, \& Breast milk. Retrieved June 30, 2021, from -

https://seattlemamadoc.seattlechildrens.org/measles -mmr-vaccine-immunity-breastmilk/

Citation: Zadran DNK., and Naibzi DZK. (2021). Incidence of measles in breastfed infants versus nonbreastfed infants. Am. J. Pure Appl. Sci., 3(5), 119-124. https://doi.org/10.34104/ajpab.021.01190124 @) 